Research Paper

\title{
Radiotherapy Alone or Concurrent Chemoradiation for Esophageal Squamous Cell Carcinoma in Elderly Patients
}

\author{
Lina Zhao ${ }^{*}$, Yongchun Zhou1 ${ }^{*}$, Haitao Pan², Yutian Yin ${ }^{1}$, Guangjin Chai ${ }^{1}$, Yunfeng Mu${ }^{1}$, Feng Xiao1, Steven \\ H. Lin ${ }^{3 凶}$, Mei Shi $^{1 凶}$ \\ 1. Department of Radiation Oncology, Xijing Hospital, Fourth Military Medical University, No. 127 West Changle Road, Xi'an, 710032 China. \\ 2. Department of Biostatistics, The University of Texas MD Anderson Cancer Center, Houston, TX, 77030; \\ 3. Department of Radiation Oncology, The University of Texas MD Anderson Cancer Center, 1515 Holcombe Blvd., Unit 097, Houston, Texas 77030, USA. \\ * Both of the authors contributed equally to this work.
}

$\square$ Corresponding authors: Mei Shi, Department of Radiation Oncology, Xijing Hospital, Fourth Military Medical University, No. 127 West Changle Road, Xi' an, 710032 China. Tel: 86-29-84775425, Fax: +86-29-83249969, Email: mshi82@hotmail.com; Steven H. Lin, Department of Radiation Oncology, The University of Texas MD Anderson Cancer Center, 1515 Holcombe Blvd., Unit 097, Houston, Texas 77030, USA. Tel: 713-563-8490, Fax: 713-563-2366, Email: shlin@mdanderson.org

(1) Ivyspring International Publisher. This is an open access article distributed under the terms of the Creative Commons Attribution (CC BY-NC) license (https:// creativecommons.org/licenses/by-nc/4.0/). See http://ivyspring.com/terms for full terms and conditions.

Received: 2017.05.02; Accepted: 2017.08.29; Published: 2017.09.15

\begin{abstract}
Purpose: The reported data of elderly ESCC are rather limited and there is a lack of information to guide treatment decisions for elderly patients with esophageal cancer. This study aims to identify the efficacy and factors for optimal treatment approaches for elderly esophageal squamous cell carcinoma (ESCC) treated with radiotherapy (RT) alone or concurrent chemoradiation (CCRT).

Methods: This study included 184 I-III elderly ESCC patients aged $\geq 70$ years treated by oral single agent CCRT (sCCRT) or double agents CCRT (dCCRT) or RT alone at a single institution in China. RT was delivered with Intensity Modulated Irradiation Therapy (IMRT) or Volumetric-Modulated Arc Therapy (VMAT). Sequential or simultaneous integrated boost (SIB) approach was applied for GTV dose escalation. Toxicities were evaluated by criteria of Radiation Therapy Oncology Group. Statistical analyses were performed on survival and failure patterns.

Results: At a median follow-up time of 15.5 months, the 2- and 3-year estimated overall survival (OS) were $43.5 \%$ and $35.2 \%$, respectively. $T$ and $N$ stage, GTV dose (cutoff value $56 \mathrm{~Gy}$ ), simultaneous integrated boost (SIB) technique and CCRT were significant predictors for the outcomes. SCCRT was significantly associated with higher OS, LRFS, and DFS when compared with RT alone and no difference was observed between sCCRT and dCCRT. 44\% patients experienced treatment failure, among whom $65.4 \%$ developed local failure. $81.3 \%$ local failure occurred in GTV and $70.6 \%$ regional failures occurred out of radiation field. dCCRT was the only independent prediction factor for grade $\geq 2$ neutropenia and gastrointestinal reactions compared with sCCRT and RT alone. No significant difference of toxicities was observed between sCCRT and RT alone.

Conclusions: Our results demonstrated that CCRT in elderly patients had significant survival benefit compared to RT alone, especially using Single oral agent. sCCRT had less toxicities compared to dCCRT, and the toxicity was similar to RT alone. GTV dose $\geq 56$ Gy and SIB technique were optimal approaches for radiotherapy.
\end{abstract}

Key words: elderly esophageal squamous cell carcinoma (ESCC), failure pattern, prognostic factor, concurrent chemoradiation (CCRT), toxicities

\section{Purpose}

According to the Cancer statistics in China of 2015[1], esophageal cancer (EC), mainly squamous carcinoma, is the third most commonly diagnosed cancer and the fourth most common cause of cancer death. $70 \%$ patients were more than 60 years and $20 \%$ patients were more than 75 years. As life expectancy is expected to increase over time in China [2], elderly esophageal cancer patients are likely to increase rapidly in the future.

Elderly patients have special characteristics 
including poor physiologic status and competing comorbidities, and therefore considerations should be taken into account including age, functional status, risk of treatment-related morbidities, life expectancy, and personal preference in making treatment decisions [3]. Due to medical comorbidities and advanced age, many elderly patients with localized esophageal squamous cell carcinoma (ESCC) are not fit for surgical resection. National comprehensive Cancer Network (NCCN) Clinical Practice Guideline has recommended 50-50.4 Gy definitive radiotherapy (RT) plus double agents chemotherapy (Fluoropyrimidine or taxane based) and the clinical tumor volume (CTV) should include coverage of elective nodal regions (Elective Nodal Irradiation, ENI) for patients unfit for surgery. However, due to underrepresentation of elderly patients in clinical trials, it is uncertain if these recommendations are fitting for patients older than 70 years of age.

Some literatures have supported that elderly patients could benefit from double agents based concurrent chemoradiotherapy (CCRT) [3-7]. Because substantially more toxicities could result from double chemotherapy agents CCRT (dCCRT) [8, 9], some studies use oral capecitabine or S-1 to replace infusional fluorouracil had shown excellent safety and efficacy results [10-12]. Single chemotherapy agents CCRT(sCCRT)with S-1 also have shown promising results [2].

However, many of these studies reflect small retrospective samples using somewhat heterogeneous treatment approaches, making them less useful in determining optimal CCRT/RT approaches. We have embarked on this study to address the definitive treatment approach in a large cohort of elderly Chinese ESCC patients aged $\geq 70$ years using modern radiotherapy approaches.

\section{Materials and methods}

\section{Patients and pretreatment evaluations}

We have included 184 consecutive elderly EC patients who have received definitive CCRT/RT in Xijing Hospital between January 2010 and December 2014. All patients were $\geq 70$ years and had ESCC, no distant metastasis at presentation and no anticancer treatment history. All cases were staged by the seventh edition of the American Joint Committee on Cancer (AJCC). Patients and treatment characteristics across different treatment modalities were summarized in Table 1. Pretreatment evaluations included blood routine and biochemistry test, barium swallow X-ray, upper endoscopy and endoscopic ultrasonography (EUS), contrast-enhanced chest and abdomen computed tomography (CT) and ultrasonography of the neck. Magnetic resonance imaging (MRI), positron emission tomographycomputed tomography (PET-CT) and bone scan were used when clinically needed. This study was approved by the ethics committee of Xijing Hospital.

\section{Radiotherapy}

Radiation therapy was delivered with IMRT or VMAT. Four-dimensional CT images were obtained in all patients with distal esophageal cancer and cases with tumor motion $>1 \mathrm{~cm}$ to allow tumor motion to be taken into consideration for treatment planning. Definition of Involved Field Irradiation (IFI) and definition of Elective Nodal Irradiation (ENI) were shown in (Supplemental figure 1A-D). The prescribed doses were 50-70 Gy to the PTV for PGTVt and PGTVnd in 25 to 35 fractions and 45-54Gy for PCTVt and PCTVnd, respectively. Sequential (dose per fraction range: 1.8-2Gy) or simultaneous integrated boost (SIB) approach (dose per fraction range: 2-2.2 Gy) was applied for GTV and GTVnd dose escalation. The treatment was delivered on consecutive weekdays with a break on intervening weekend days if applicable.

\section{Chemotherapy regimes}

$47.7 \%$ patients received sCCRT including capecitabine or S1 alone, and dCCRT including cisplatin plus capecitabine or S1. The choice of chemotherapy was based on multidisciplinary team decision and patients' preference. No patients received induction or adjuvant chemotherapy.

\section{Follow-up evaluations}

All patients were followed up every 3 months in the first 2 years and every 6 months thereafter. Each follow-up included a physical examination, routine blood count and chemistries, barium swallow, ultrasonography of neck and abdomen, and contrast enhanced CT scans of the neck and thorax. Endoscopy with or without biopsy was recommended every 6 months or as clinically indicated. Radiotherapyrelated toxicities were evaluated and scored based on the scoring criteria of the Radiation Therapy Oncology Group.

\section{Definition of treatment failure}

Treatment failure was determined by pathological evidence or progression in serial posttreatment images including barium swallow, CT scans, PET-CT scans, and endoscopic evaluations. Local and regional failure was defined as the persistence or recurrence of the primary tumor or regional lymph nodes, respectively. Distant metastatic failure was defined as metastasis to any site beyond the primary tumor and regional lymph nodes. Failure 
location (PGTV, PCTV, or outside the radiation field)

of the treatment plan.

was identified by fusing current image with CT scan

Table 1. Patient and treatment characteristics of elderly ESCC patients across different treatment modalities.

\begin{tabular}{|c|c|c|c|c|c|}
\hline \multirow[t]{2}{*}{ Characteristics } & \multicolumn{3}{|c|}{ No. of patients (\%) } & \multirow[t]{2}{*}{$x^{2}$} & \multirow[t]{2}{*}{$\mathrm{P}$} \\
\hline & RT alone & Single agent CRT & Double agent CRT & & \\
\hline Age (years) & & & & 29.898 & $<0.001 \mathrm{a}$ \\
\hline$<75$ & $27(27.6 \%)$ & $38(54.3 \%)$ & $15(93.8 \%)$ & & \\
\hline$\geq 75$ & $71(72.4 \%)$ & $32(45.7 \%)$ & $1(6.2 \%)$ & & \\
\hline Sex & & & & 6.692 & $0.035 \mathrm{~b}$ \\
\hline Male & $54(54.5 \%)$ & $52(74.3 \%)$ & $11(68.8 \%)$ & & \\
\hline Female & $44(45.5 \%)$ & $18(25.7 \%)$ & $5(31.2 \%)$ & & \\
\hline Smoking (pack years) & & & & 4.745 & 0.093 \\
\hline$<20$ & $74(74.7 \%)$ & $47(67.1 \%)$ & $8(50.0 \%)$ & & \\
\hline$\geq 20$ & $24(25.3 \%)$ & $23(32.9 \%)$ & $8(50.0 \%)$ & & \\
\hline Alcohol & & & & 3.564 & 0.168 \\
\hline Not heavy drinking & $92(92.9 \%)$ & $61(87.1 \%)$ & $13(81.3 \%)$ & & \\
\hline Heavy drinking & $6(7.1 \%)$ & $9(12.9 \%)$ & $3(18.7 \%)$ & & \\
\hline Comorbidities & & & & 1.240 & 0.538 \\
\hline No & $55(55.6 \%)$ & $44(62.9 \%)$ & $8(50.0 \%)$ & & \\
\hline Yes & $43(44.4 \%)$ & $26(37.1 \%)$ & $8(50.0 \%)$ & & \\
\hline ECOG performance status & & & & 4.285 & 0.117 \\
\hline $0-1$ & $85(85.9 \%)$ & $63(90.0 \%)$ & $16(100.0 \%)$ & & \\
\hline $2-3$ & $13(14.1 \%)$ & $7(10.0 \%)$ & $0(0.0 \%)$ & & \\
\hline Weight loss before therapy & & & & 6.019 & $0.049 b$ \\
\hline$<5 \%$ & $55(55.6 \%)$ & $52(74.3 \%)$ & $11(68.8 \%)$ & & \\
\hline$\geq 5 \%$ & $43(44.4 \%)$ & $18(25.7 \%)$ & $5(31.2 \%)$ & & \\
\hline Weight loss during therapy & & & & 4.727 & 0.094 \\
\hline$<5 \%$ & $73(73.7 \%)$ & $61(87.1 \%)$ & $13(81.3 \%)$ & & \\
\hline$\geq 5 \%$ & $26(26.3 \%)$ & $9(12.9 \%)$ & $3(18.7 \%)$ & & \\
\hline Primary tumor location & & & & 0.581 & 0.748 \\
\hline Upper-middle & $47(47.5 \%)$ & $32(45.7 \%)$ & $9(56.3 \%)$ & & \\
\hline Middle-lower & $51(52.5 \%)$ & $38(54.3 \%)$ & $7(43.7 \%)$ & & \\
\hline Primary tumor length & & & & 0.619 & 0.734 \\
\hline$<6.5 \mathrm{~cm}$ & $33(33.3 \%)$ & $25(35.7 \%)$ & $7(43.7 \%)$ & & \\
\hline$\geq 6.5 \mathrm{~cm}$ & $65(66.7 \%)$ & $45(64.3 \%)$ & $9(56.3 \%)$ & & \\
\hline AJCC stage & & & & 3.034 & 0.219 \\
\hline I-II & $17(17.2 \%)$ & $20(28.6 \%)$ & $4(25.0 \%)$ & & \\
\hline III & $81(82.8 \%)$ & $50(71.4 \%)$ & $12(75.0 \%)$ & & \\
\hline T stage & & & & 3.462 & 0.177 \\
\hline $1-2$ & $12(12.1 \%)$ & $16(22.9 \%)$ & $2(12.5 \%)$ & & \\
\hline $3-4$ & $86(87.9 \%)$ & $54(77.1 \%)$ & $14(87.5 \%)$ & & \\
\hline N stage & & & & 4.646 & 0.098 \\
\hline $1-2$ & $51(52.5 \%)$ & $48(68.6 \%)$ & $9(56.3 \%)$ & & \\
\hline $3-4$ & $47(47.5 \%)$ & $22(31.4 \%)$ & $7(43.7 \%)$ & & \\
\hline Radiotherapy technique & & & & 2.095 & 0.351 \\
\hline IMRT & $60(60.6 \%)$ & $39(55.7 \%)$ & $12(75.0 \%)$ & & \\
\hline VMAT & $38(39.4 \%)$ & $31(44.3 \%)$ & $4(25.0 \%)$ & & \\
\hline CTVnd delineation & & & & 2.150 & 0.341 \\
\hline IFI & $72(72.7 \%)$ & $58(82.9 \%)$ & $12(75.0 \%)$ & & \\
\hline ENI & $26(27.3 \%)$ & $12(17.1 \%)$ & $4(25.0 \%)$ & & \\
\hline Dose boost schemes & & & & 2.611 & 0.271 \\
\hline No SIB & $34(34.3 \%)$ & $28(40.0 \%)$ & $3(18.7 \%)$ & & \\
\hline SIB & $64(65.7 \%)$ & $42(60.0 \%)$ & $13(81.3 \%)$ & & \\
\hline Fraction dose (Gy) & & & & 0.917 & 0.632 \\
\hline$\leq 2$ & $57(57.6 \%)$ & $36(51.4 \%)$ & $8(50.0 \%)$ & & \\
\hline$>2$ & $41(42.4 \%)$ & $34(48.6 \%)$ & $8(50.0 \%)$ & & \\
\hline Radiation dose for GTV (Gy) & & & & 1.208 & 0.547 \\
\hline$<56$ & $15(15.2 \%)$ & $11(15.7 \%)$ & $1(6.2 \%)$ & & \\
\hline$\geq 56$ & $83(84.8 \%)$ & $59(84.3 \%)$ & $15(93.8 \%)$ & & \\
\hline Radiation dose for CTV (Gy) & & & & 2.315 & 0.314 \\
\hline$<50$ & $17(17.2 \%)$ & $10(14.3 \%)$ & $5(31.2 \%)$ & & \\
\hline$\geq 50$ & $81(82.8 \%)$ & $60(85.7 \%)$ & $11(68.8 \%)$ & & \\
\hline $\begin{array}{l}\text { Abbreviations: ECOG, eastern c } \\
\text { CTV: clinical target volume; IFI, } \\
p \text { values less than } 0.05 \text { are highli } \\
\text { a There were significant differen } \\
\text { b There were significant differen }\end{array}$ & $\begin{array}{l}\text { gy group; IN } \\
\text { radiation; EN } \\
\text { ee groups. }\end{array}$ & $\begin{array}{l}\text { odulated radiother } \\
\text { irradiation; SIB, sin }\end{array}$ & $\begin{array}{l}\text { volumetric-modulate } \\
\text { tegrated boost. }\end{array}$ & & \\
\hline
\end{tabular}




\section{Statistical analysis}

All radiation doses were converted into the equivalent dose in 2 Gy fraction (EQD2), using an $\alpha / \beta=10$ and calculated using the prescribed dose $\times(10+$ dose per fraction $) / 12$. Distant failure was defined as the metastasis beyond the primary tumor and regional lymph nodes station. Overall survival (OS), loco-regional failure-free survival (LRFFS), distance metastasis free survival (DMFS), and progression-free survival (PFS), were defined as the time from the first date of therapy until the date of death, local-regional failure, distant metastasis and tumor progression respectively. And they were calculated by Kaplan-Meier method. The log-rank tests were also used for univariate analyses to select potential prognostic factors. We used $p$ value less than 0.10 as cutoff to screen the factors for the subsequent multivariate analyses. Cox proportional hazard model was used for multivariate analysis. Continuous variables were dichotomized by cut-off values which were identified by receiver operating characteristic curves or based on clinical significance. Chi-square tests, Univariate and multivariate logistic regression analyses were also conducted to study any possible differences of treatment-related toxicities. All statistical analyses were performed using SPSS 18.0 (SPSS, Chicago, IL, USA) software.

\section{Results}

\section{Patients' characteristics}

Patient and treatment characteristics across three different treatment modalities were mostly well balanced, except that younger patients tended to get dCCRT or sCCRT, male patients and those with weight loss $<5.0 \%$ before therapy tended to receive sCCRT compared with RT alone (Table 1). The median age was 76 years (range, $70-91$ ). $71.3 \%$ had stage III disease. The patients were treated by either IMRT or VMAT. The median EQD2 dosefor GTV was 61.6 Gy (range, 50-70 Gy).

\section{Treatment efficacy}

The median follow-up time was 15.5 months (interquartile range 8.0-25.4 months), The 2- and 3-year estimated OS, LRFFS, DMFS and PFS were $43.5 \%, 60.5 \%, 79.9 \%$ and $34.6 \%$, and $35.2 \%, 58.5 \%$, $77.8 \%$ and $29.4 \%$, respectively (Figure 1 ). A total of 72 patients $(39.1 \%)$ were alive at the end of the study period with a median follow-up duration of 24.0 months (interquartile range 18.8-38.5months). Median OS time was 18.6 months in this study.

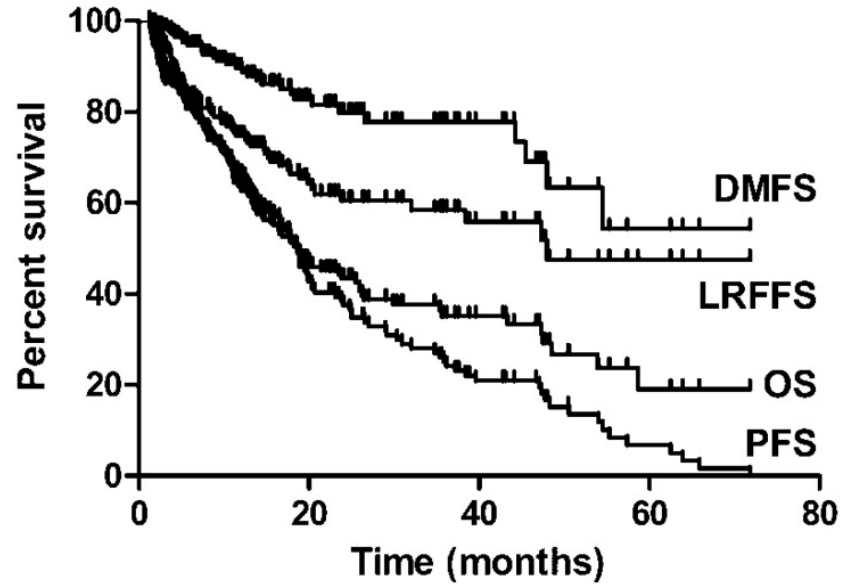

Figure 1. OS, LRFFS, DMFS and PFSfor elderly ESCC.

\section{Prognostic analysis}

Results of the univariate analyses for screening potential significant factors were shown in Supplement Table 1. Of these factors, concurrent chemotherapy led to significant better OS and PFS ( $p$ value $<0.001)$. Although no significant difference was found between SCCRT and dCCRT in relation to outcomes, sCCRT was significantly associated with higher OS, LRFS, and DFS when compared with RT alone, which was shown in Figure 2.

Multivariate analyses showed that $\mathrm{T}$ stage, $\mathrm{N}$ stage, GTV EQD2 dose (cutoff value 56Gy) and CCRT were significant predictors for OS, $\mathrm{N}$ stage and CCRT were significant predictors for LRFFS, $\mathrm{N}$ stage was also predictive of DMFS and $\mathrm{T}$ stage, $\mathrm{N}$ stage, SIB technique and CCRT could predict PFS significantly (table 2).

\section{Failure patterns and salvage treatment}

Failure pattern analysis and representable cases of recurrence were shown in Figure 3. Cumulatively, 81 patients (44\%) experienced treatment failure and 47 $(58 \%)$ failures were biopsy proven. The median time to any failure was 7.1 months (range 1.3-48.0 months). $53(65.4 \%)$ and $17(21.0 \%)$ patients had developed local and regional failure respectively. 8 (9.8\%) patients developed both local and regional failures. The median time to local and regional failure was 11.0 months (range 1.9-53.9 months) and 8.8 months (range 1.5-20.7 months), respectively. $81.3 \%$ local failure occurred in PGTVt (43/53), and the others were located in PCTV $(9.4 \%, 5 / 53)$ and out of field $(9.4 \%, 5 / 53)$, respectively. $70.6 \%$ occurred out of radiation field (12/17), among whom $91.7 \%$ received IFI (11/12). The other regional failures were located in PGTVnd $(5.9 \%, 1 / 17)$ and PCTV $(23.5 \%, 4 / 17)$ respectively. sCCRT has significantly higher LRFFS compared with RT alone ( $69.6 \%$ vs $52 \%$, p $=0.05)$. 
Distant metastasis was noted in $30(37.0 \%)$ patients. The median time to distant metastasis was 10.9 months (range 1.6-54.5 months). The most common metastasis sites were lung $(12 / 30,40.0 \%)$, liver $(12 / 30$, $40.0 \%)$, bone $(8 / 30,26.7 \%)$ and lower abdomen lymph nodes $(2 / 30,6.7 \%)$, and $4(13.3 \%)$ patients had multiple metastases.

Among 81 patients with treatment failure, 9 patients $(11.1 \%)$ received salvage or palliative radiotherapy, among whom 2 received irradiation to the esophageal lesion, 4 received irradiation to the regional lymph nodes, 1 received irradiation to both esophageal lesion and regional lymph nodes and 1 received irradiation to the regional lymph nodes and stereotactic body radiation therapy to the oligometastatic lesions of the lung. At the end of the study period, 3 patients were still alive and the mean survival time for these patients were 26 months.

Table 2. Multivariateanalysis of prognostic factorson treatment resultsfor elderly EC.

\begin{tabular}{|c|c|c|c|}
\hline \multirow[t]{2}{*}{ Endpoint } & \multirow[t]{2}{*}{ Prognostic factors } & \multicolumn{2}{|c|}{ Multivariateanalysis } \\
\hline & & $P$ & HR $(95 \% C I)$ \\
\hline \multirow[t]{4}{*}{ 3y OS } & T stage (1-2vs3-4) & 0.039 & $2.205(1.041-4.672)$ \\
\hline & $\mathrm{N}$ stage $(0-1 v s 2-3)$ & $<0.001$ & $3.093(1.861-5.140)$ \\
\hline & GTV dose $(<56 \mathrm{~Gy} v s \geq 56 \mathrm{~Gy})$ & 0.020 & $0.475(0.254-0.889)$ \\
\hline & Concurrent chemotherapy (No vs Yes) & 0.001 & $0.413(0.249-0.686)$ \\
\hline \multirow[t]{2}{*}{ 3y LRFFS } & N stage $(0-1 v s 2-3)$ & $<0.001$ & $3.815(1.959-7.432)$ \\
\hline & Concurrent chemotherapy (No vs Yes) & 0.033 & $0.489(0.254-0.943)$ \\
\hline 3y DMFS & N stage $(0-1 v s 2-3)$ & 0.035 & $2.252(1.060-4.782)$ \\
\hline \multirow[t]{4}{*}{ 3y PFS } & T stage (1-2vs3-4) & 0.028 & $1.971(1.076-3.613)$ \\
\hline & $\mathrm{N}$ stage $(0-1 v s 2-3)$ & $<0.001$ & $2.642(1.829-3.816)$ \\
\hline & Dose boost schemes (sequential boost vs SIB) & 0.010 & $0.608(0.416-0.889)$ \\
\hline & Concurrent chemotherapy (No vs Yes) & 0.001 & $0.537(0.370-0.780)$ \\
\hline
\end{tabular}

Abbreviations: OS, overall survival; LRFFS, local-regional failure-free survival; DMFS, distance metastasis free survival; PFS, progression-free survival; HR, hazard ratio; 95\% CI, $95 \%$ confidence interval.

A

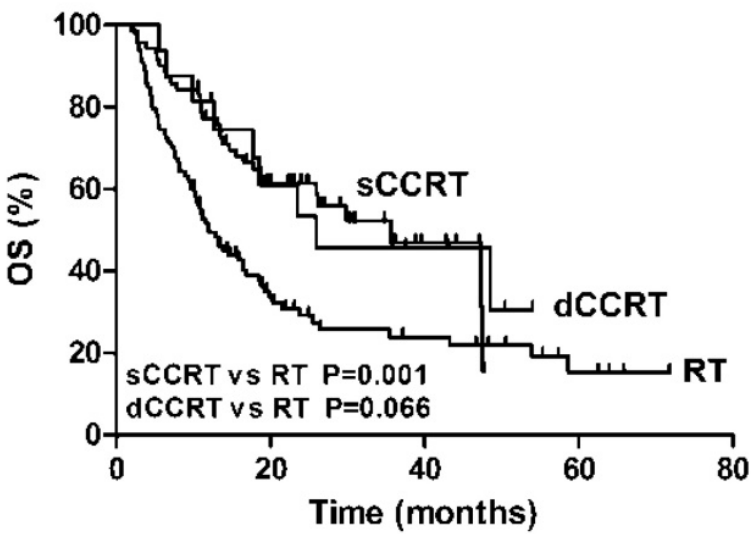

C

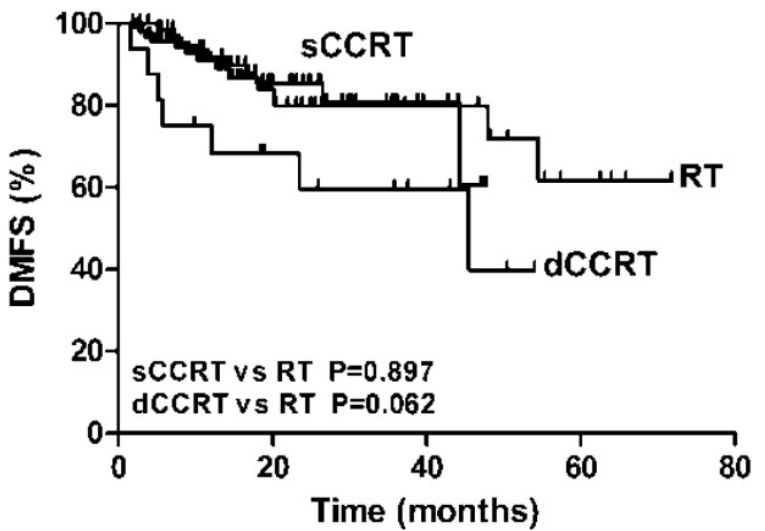

B

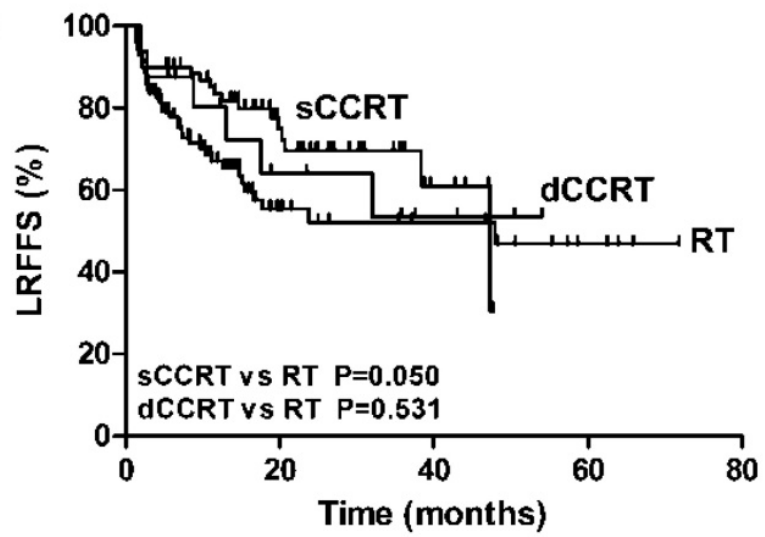

D

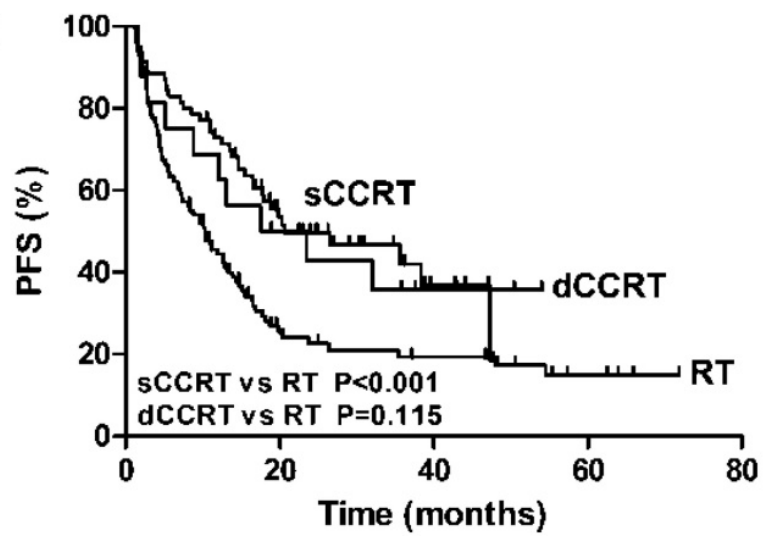

Figure 2. The association of RT alone, sCCRT anddCCRTwith OS (A), LRFFS (B), DMFS (C) and PFS (D) of elderly ESCC. 
Table 3. Treatment related toxicities.

\begin{tabular}{|c|c|c|c|c|c|c|c|c|}
\hline \multirow[b]{2}{*}{ Toxicities } & \multicolumn{2}{|l|}{ RT alone } & \multicolumn{2}{|c|}{ Single agent CRT } & \multicolumn{2}{|c|}{ Double agents' CRT } & \multirow[t]{2}{*}{$x^{2}$} & \multirow[t]{2}{*}{$\mathrm{P}$} \\
\hline & Grade $0-1$ & $\geq$ Grade 2 & Grade $0-1$ & $\geq$ Grade 2 & Grade $0-1$ & $\geq$ Grade 2 & & \\
\hline Neutropenia & $77(78.6)$ & $21(21.4)$ & $53(75.7)$ & $17(24.3)$ & $6(37.5)$ & $10(62.5)$ & 10.602 & $0.005 \mathrm{a}$ \\
\hline Thrombocytopenia & $93(94.9)$ & $5(5.1)$ & $62(88.6)$ & $8(11.4)$ & $13(81.2)$ & $3(18.8)$ & 4.030 & 0.133 \\
\hline Radiation esophagitis & $79(80.6)$ & 19(19.4) & $59(83.3)$ & $11(15.7)$ & $11(68.8)$ & $5(31.2)$ & 1.893 & 0.388 \\
\hline Radiation pneumonia & $91(92.9)$ & $7(7.1)$ & $67(95.7)$ & $3(4.3)$ & $15(93.8)$ & $1(6.2)$ & 0.619 & 0.734 \\
\hline Gastrointestinal reaction & $94(95.9)$ & $4(4.1)$ & $66(94.3)$ & $4(5.7)$ & $6(37.5)$ & $10(62.5)$ & 32.605 & $<0.001 b$ \\
\hline
\end{tabular}

$\mathrm{p}$ values less than 0.05 were highlighted in bold.

a: Double agentsvs Single agent CRT, $\mathrm{P}=0.003$; Double agents' ${ }^{\prime}$ CRT VS RT alone, $\mathrm{P}=0.002$

b: Double agents VS Single agent CRT, P < 0.001; Double agents' CRT VS RT alone, $\mathrm{P}<0.001$

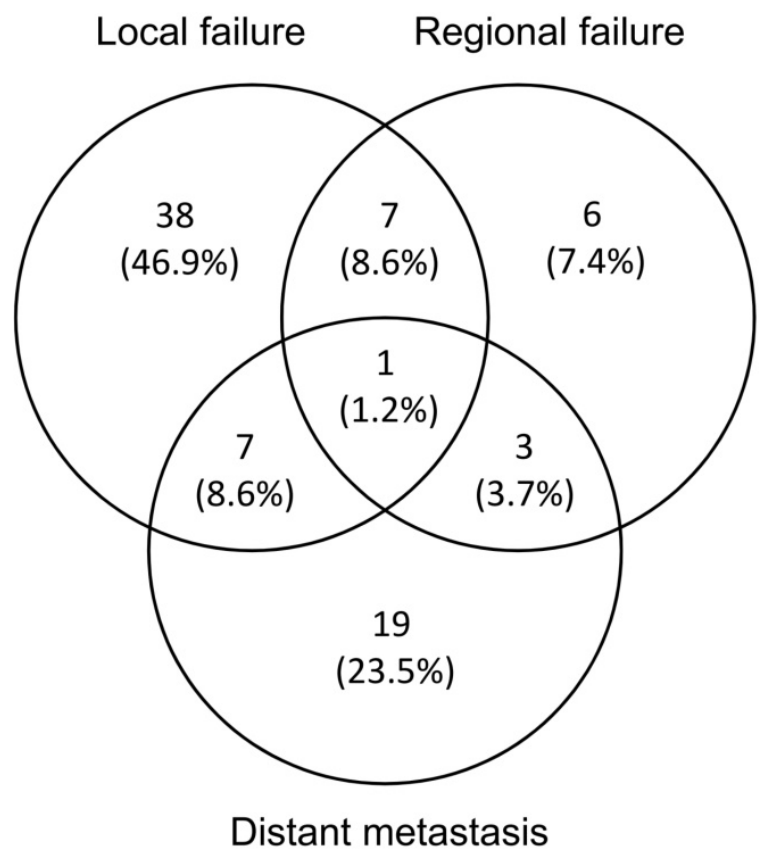

Figure 3. Failure pattern analysis

\section{Toxicities}

The most commonly observed acute toxicity included radiation esophagitis, radiation pneumonitis, thrombocytopenia, leucopenia and gastrointestinal reactions, which were mainly mild or asymptomatic for RT alone and sCCRT group (grade $0-1)$. For CCRT group, the incidence of Grade $\geq 2$ neutropenia $(62.5 \%$ vs $37.5 \%)$ and gastrointestinal reactions $(62.5 \%$ vs $37.5 \%)$ were slightly higher when compared with grade 0-1 toxicities. There were no treatment-related deaths. For the whole group, the incidence of grade 3-4 esophagitis, leucopenia, thrombocytopenia, nausea and vomiting and radiation pneumonitis were $1.6 \%(3 / 184), 6.0 \%$ $(11 / 184,1$ for grade 4$), 2.7 \%(5 / 184,1$ for grade 4$)$, $1.1 \%(2 / 184)$ and $1.6 \%(3 / 184)$, respectively.

There was no significant difference among three treatment groups with respect to Grade $0-1$ toxicities $(p=0.580)$. However, patients treated with dCCRT developed significantly more grade $\geq 2$ neutropenia (dCCRT vs sCCRT: $62.5 \%$ vs $24.3 \%, p=0.003$; dCCRT vs RT alone: $62.5 \%$ vs $21.4 \%, p=0.002)$ and gastrointestinal reactions (dCCRT vs sCCRT: $62.5 \%$ vs $5.7 \%, p<0.001$; dCCRT vs RT alone: $62.5 \%$ vs $4.1 \%$, $p<0.001)$ than patients who received sCCRT and RT alone. Although grade $\geq 2$ thrombocytopenia in sCCRT was higher than that of RT alone $(11.4 \%$ vs $5.1 \%)$, no significant difference was observed between these two treatment modalities (Table 3). Furthermore, we did logistic regression analysis and found dCCRT was the only independent prediction factor for grade $\geq 2$ neutropenia and gastrointestinal reactions. All the other factors, including radiation dose and treatment volumes, could not show effect on the toxicities (supplementary table 1 and 2).

\section{Discussion}

This is a large single-institution series which focused on 184 stage 1-IIIESCC patients aged $\geq 70$ years treated with definitive CCRT or RT alone. Our results confirmed that CCRT had significant survival benefit compared to RT alone, with similar efficacy whether chemotherapy was administered as single or double agents. Although the tradeoff of increased acute toxicities of concurrent chemotherapy was seen, sCCRT had less toxicities compared to dCCRT, and the toxicity was nearly equivalent to RT alone. These data suggest that for elderly ESCC patients, sCCRT is likely the optimal approach.

The role of CCRT in elderly EC patients remained unclear. Due to poor physiologic function, medical comorbidities, advanced age, or patient preference, many older patients with localized esophageal cancer are not candidates for surgical resection [3]. Definitive chemoradiation has been established by the landmark RTOG 85-01 trial[13], demonstrating that the addition of concurrent cisplatin-based chemotherapy to conventional fractionation RT was associated with a significantly better median survival (14 versus 9 months) and five-year survival (27 versus 0 percent) than RT alone. NCCN have recommended 50-50.4 Gy RT plus concurrent double agents chemotherapy (Fluoropyrimidine or taxane based) for EC patients unfit for surgery. For elderly EC, the results of RT alone or CCRT were sporadically reported. Xu et al 
reported EC patients aged $>/=70$ years treated with RT alone and the 3- and 5-year OS were 13.2 and 9.2\%, respectively [14]. As the results were not very satisfactory compared with reported CCRT results, they suggested RT alone for elderly patients with EC did not appear to be acceptable. Another study found ESCC patients aged $>70$ years treated by CCRT had improved survival rates compared with RT alone, with the overall and progression-free survival in the CRT group versus the RT group were 17 months versus eight months $(P=0.013)$ and 14 months versus five months $(P=0.01)$, respectively[9]. Zhang et al also indicated that he 3-year OS rate was 36.1\% for CCRT compared with $28.5 \%$ following RT alone $(p=0.008)$. Multivariate analysis revealed that survival was significantly better in the CCRT group than RT alone group for patients $</=72$ years [5]. Our study also indicated that CCRT was a significant prognostic indicator for OS, LRFFS and PFS in elderly patients. These results suggested the value of CCRT in elderly EC patients still existed.

Although promising results were shown for CCRT in elderly patients, it has to be noted that more severe toxicities were related toCCRT compared with RT alone in elderly EC, especially for dCCRT. Cui et al found CCRT was one of the independent factors for radiation pneumonitis[15].Xu et al reported acute grade 3-4 esophagitis in 5.4\% CCRT group and $2.7 \%$ RT group, respectively[9]. Zhang et al found that acute grade 3-4 hematological toxicity was identified in $36.9 \%$ and $14.5 \%$ in CCRT and RT alone patients, respectively $(p=0.001)$. CCRT patients had more grade $\geq 2$ esophagitis and pneumonitis than RT alone $(52.1 \%$ vs. $34.5 \%, p=0.005)[5]$.We also found that patients treated with dCCRT developed significantly more grade $\geq 2$ neutropenia and gastrointestinal reactions than patients who received sCCRT and RT alone. However, severe (grade 3 and 4) esophagitis was very rare $(<2 \%)$ and grade $3-4$ hematological toxicity was less than $10 \%$ in our study, which might be due to advanced RT technique and optimal treatment plan utilized, furthermore, we use oral capecitabine or S-1 to replaceinfusional fluorouracil in double agents regimes, which was also recommended by NCCN guideline and has been shown to be effective in some previous studies [10-12]. These results suggested that dCCRT should be given to patients with caution and should be used in highly selected elderly patients.

Owing to the toxicities of dCCRT, sCCRT attracted more and more attention aiming at decreasing the toxicities without compensating efficacies. A phase I trial reported oral S-1 and RT in the treatment of elderly EC patients. No grade 3 or 4 toxicity was observed at 60 and $70 \mathrm{mg} / \mathrm{m} 2$ dose levels. Endoscopic complete responsewas 66.7\% andmedian OS was 29 months [2]. In our study, 38\% patients received concurrent oral capecitabine or S-1 with RT. Our results showed that although no significant difference was found between SCCRT and dCCRTin relate to OS, both SCCRT and dCCRT were significantly associated with higher OS rate compared with RT alone. Furthermore, we observed significant less toxicities of sCCRT when compared with dCCRT and no significant difference in toxicities were observed between sCCRT and RT alone. These results strongly suggested that oral capecitabine or S-1 could be considered as an option for elderly patients who were unable to tolerate the infusional chemotherapy.

There is no consensus on the optimal RT design and delivery, especially for elderly ESCC. With the advance of RT technique from 2D to 3D, from 3DCRT to IMRT and VMAT, the survival outcome has been remarkably improved. Our results showed that CCRT/RT in elderly patients produced an excellent overall survival, with the 2- and 3-year estimated OS, LRFFS, DMFS and PFS were $43.5 \%, 60.5 \%, 79.9 \%$ and $34.6 \%$, and $35.2 \%, 58.5 \%, 77.8 \%$ and $29.4 \%$, respectively and the median OS time was 18.6 months in this study. These results were comparable with or slightly better than previous studies [4, 5, 9, 11, 16-19], which is in part due to the application VMAT and IMRT for RT.

The optimal CTVnd delineation method and RT volume remained unclear in the treatment of ESCC. Liu et al studied 169 elderly EC patients receiving IFI and ENI and the results showed that no significance difference was seen for OS and in-field recurrence and distal organ metastasis between the two groups [20]. Our results also showed that no significant difference was found between IFI and ENI in relation to the reported outcomes and toxicities in these studies. However, we did found among 17 patients with regional failures, $70.6 \%$ occurred out of radiation field (12/17), among whom $91.7 \%$ received IFI (11/12) and ENI method was borderline significantly associated with regional failure free survival $(p=0.062)$, indicating ENI might be related to better regional control but could not translated to OS.

The optimal dose for ESCC was still controversial and 50.4Gy remained the standard [21]. Some studies showed that higher dose RT could generate excellent outcomes. One study indicated that the results of 66Gy irradiation alone were comparable to those in younger patients and the median survival time and overall survival rate at 3 years was 30 months and 39\%, respectively ${ }^{7}$.Xu et al indicated that patients irradiated with a dose of $>50$ Gy plus chemotherapy had a significant improved survival compared with those who receiving a dose of $</=50$ 
Gy plus chemotherapy (18 vs. 14 months; $P=0.049$ ) [9]. We also found the GTV EQD2 dose $\geq 56$ Gy was significantly associated with higher OS, DMFS and PFS ( $p$ value $<0.05)$ and GTV EQD2 dose was an independent indicator for OS $(P=0.020, \mathrm{HR}=0.475)$. However, CTV EQD2 dose (50Gy as cutoff) was not significantly related with the reported outcomes and also the regional control rate $(72.6 \%$ vs $87.3 \%$, $P=0.384$ ) in this study. These results suggested the potential benefit of escalating GTV dose which was also confirmed by our results showing OS, LRFFS and PFS of patients using SIB technique to boost GTV dose and higher fraction dose ( $>2 \mathrm{~Gy}$ ) were both significantly higher than those using sequential boost technique and lower fraction dose ( $\leq 2$ Gy) ( $p$ value $<0.05)$. The failure pattern analysis also indicated that $65.4 \%$ patients had developed local failure and $81.3 \%$ local failure occurred in PGTVt, suggesting GTV should be prescribed a relatively higher dose based on precise target delineation and rigorous quality control as well as a careful evaluation of treatment plan to balance the benefits and toxicities.

Due to the retrospective nature, this study was limited by the potential confounding factors and the single institutional nature may also limit the applicability of our findings. Therefore, well-designed, larger multi-center prospective trials may be needed. Furthermore, detailed analysis of the nutrition status and late toxicities in elderly ESCC need to be performed in future studies.

\section{Conclusion}

Our results confirmed that CCRT in elderly patients aged $\geq 70$ years had significant survival benefit compared to RT alone by using modern RT techniques, especially using Single oral agent. sCCRT had substantially less toxicities compared to dCCRT, and the toxicity was equivalent to RT alone. GTV dose $\geq 56$ Gy and SIB technique were good options for definitive treatment of elderly ESCC patients.

\section{Abbreviations}

ESCC: esophageal squamous cell carcinoma; RT: radiotherapy; CCRT: concurrent chemoradiation; sCCRT: single agent CCRT; dCCRT: double agents CCRT; IMRT: Intensity Modulated Irradiation Therapy; VMAT: Volumetric-Modulated Arc Therapy; SIB: Sequential or simultaneous integrated boost; EC: esophageal cancer; NCCN: National comprehensive Cancer Network; GTV: Gross tumor volume; CTV: clinical tumor volume; ENI: Elective Nodal Irradiation; AJCC: American Joint Committee on Cancer; EUS: endoscopic ultrasonography; CT: computed tomography; MRI: Magnetic resonance imaging; PET-CT: positron emission tomography-computed tomography; IFI: Involved Field Irradiation; ENI: Elective Nodal Irradiation; EQD2: equivalent dose in 2 Gy fraction; OS: Overall survival; LRFFS: loco-regional failure-free survival; DMFS: distance metastasis free survival; PFS: progression-free survival.

\section{Supplementary Material}

Supplementary figures and tables. http://www.jcancer.org/v08p3242s1.pdf

\section{Acknowledgements}

This work was supported by National Natural Science Foundation of China Grants 81101689 and 81372421.

\section{Availability of data and materials}

Materials in the manuscript are available by contacting the author at zhaolinazln@outlook.ocm

\section{Authors' contributions}

Drs. Mei Shi, and Steven H. Lin were principal investigators and contributed to the study design and approval of the final version of the manuscript. Drs. Lina zhao and Yongchun Zhou contributed to manuscript writing and data interpretation. Drs. Haitao Pan and Yutian Yin contributed to data analysis. Drs. Guangjin Chai, Yunfeng Mu and Feng Xiao contributed to data collection. All authors read and approved the final manuscript.

\section{Competing Interests}

The authors have declared that no competing interest exists.

\section{References}

1. Chen W, Zheng R, Baade PD, Zhang S, Zeng H, Bray F, et al. Cancer statistics in China, 2015. CA Cancer J Clin. 2016; 66: 115-32.

2. Ji Y, Qiu G, Sheng L, Sun X, Zheng Y, Chen M, et al. A phase I dose escalation study of S-1 with concurrent radiotherapy in elderly patients with esophageal cancer. J Thorac Dis. 2016; 8: 451-8.

3. Won E, Ilson DH. Management of localized esophageal cancer in the older patient. Oncologist. 2014; 19: 367-74.

4. Song $\mathrm{T}$, Zhang $\mathrm{X}$, Fang $\mathrm{M}$, Wu S Concurrent chemoradiotherapy using paclitaxel plus cisplatin in the treatment of elderly patients with esophageal cancer. Onco Targets Ther. 2015; 8: 3087-94.

5. Zhang P, Xi M, Zhao L, Shen JX, Li QQ, He LR, et al. Is there a benefit in receiving concurrent chemoradiotherapy for elderly patients with inoperable thoracic esophageal squamous cell carcinoma? PLoS One. 2014; 9: e105270.

6. Kawashima M, Kagami Y, Toita T, Uno T, Sugiyama M, Tamura Y, et al. Prospective trial of radiotherapy for patients 80 years of age or older with squamous cell carcinoma of the thoracic esophagus. Int J Radiat Oncol Biol Phys. 2006; 64: 1112-21.

7. Tougeron D, Di Fiore F, Thureau S, Berbera N, Iwanicki-Caron I, Hamidou $\mathrm{H}$ et al. Safety and outcome of definitive chemoradiotherapy in elderly patients with oesophageal cancer. Br J Cancer. 2008; 99: 1586-92.

8. Takeuchi S, Ohtsu A, Doi T, Kojima T, Minashi K, Mera K, et al. A retrospective study of definitive chemoradiotherapy for elderly patients with esophageal cancer. Am J Clin Oncol. 2007; 30: 607-11.

9. Xu HY, Du ZD, Zhou L, Yu M, Ding ZY, Lu Y. Safety and efficacy of radiation and chemoradiation in patients over 70 years old with inoperable esophageal squamous cell carcinoma. Oncol Lett. 2014; 7: 260-6.

10. Iwase H, Shimada M, Tsuzuki T, Hirashima N, Okeya M, Hibino $Y$, et al. Concurrent chemoradiotherapy with a novel fluoropyrimidine, S-1, and 
cisplatin for locally advanced esophageal cancer: long-term results of a phase II trial. Oncology. 2013; 84: 342-9.

11. Xing L, Liang $\mathrm{Y}$, Zhang J, Wu P, Xu D, Liu F, et al. Definitive chemoradiotherapy with capecitabine and cisplatin for elder patients with locally advanced squamous cell esophageal cancer. J Cancer Res Clin Oncol. 2014; 140: 867-72.

12. Lee SJ, Ahn BM, Kim JG, Sohn SK, Chae YS, Moon JH, et al. Definitive chemoradiotherapy with capecitabine and cisplatin in patients with esophageal cancer: a pilot study. J Korean Med Sci. 2009; 24: 120-5.

13. Herskovic A, Martz K, al-Sarraf M, Leichman L, Brindle J, Vaitkevicius V, et al. Combined chemotherapy and radiotherapy compared with radiotherapy alone in patients with cancer of the esophagus. N Engl J Med. 1992; 326: 1593-8.

14. $\mathrm{Lu} \mathrm{X}, \mathrm{Wu} \mathrm{H}$, Wang J, Chen $\mathrm{Y}, \mathrm{Xu}$ J. Factors affecting long-term survival in elderly patients undergoing radiotherapy for esophageal carcinoma. Mol Clin Oncol. 2014; 2: 571-4.

15. Cui Z, Tian Y, He B, Li H, Li D, Liu J, et al. Associated factors of radiation pneumonitis induced by precise radiotherapy in 186 elderly patients with esophageal cancer. Int J Clin Exp Med. 2015; 8: 16646-51.

16. Servagi-Vernat S, Crehange G, Roullet B, Guimas V, Maingon P, Puyraveau M, et al. Phase II Study of a Platinum-Based Adapted Chemotherapy Regimen Combined with Radiotherapy in Patients 75 Years and Older with Esophageal Cancer. Drugs Aging. 2015; 32: 487-93.

17. Guo $\mathrm{JH}$, Chen $\mathrm{MQ}$ Chen $\mathrm{C}, \mathrm{Lu} \mathrm{HJ}, \mathrm{Xu} B \mathrm{BH}$. Efficacy and toxicity of nimotuzumab combined with radiotherapy in elderly patients with esophageal squamous cell carcinoma. Mol Clin Oncol. 2015; 3: 1135-8.

18. $\mathrm{Lu} \mathrm{X}, \mathrm{Wu} \mathrm{H}$, Wang J, Xu J. Short- and long-term outcomes of definitive chemoradiotherapy in patients with esophageal carcinoma aged $>/=75$ years. Mol Clin Oncol. 2014; 2: 297-301.

19. Wakui R, Yamashita H, Okuma K, Kobayashi S, Shiraishi K, Terahara A, et al. Esophageal cancer: definitive chemoradiotherapy for elderly patients. Dis Esophagus. 2010; 23: 572-9.

20. Liu M, Zhao K, Chen Y, Jiang GL. Evaluation of the value of ENI in radiotherapy for cervical and upper thoracic esophageal cancer: a retrospective analysis. Radiat Oncol. 2014; 9: 232

21. Ajani JA, D'Amico TA, Almhanna K, Bentrem DJ, Besh S, Chao J, et al. Esophageal and esophagogastric junction cancers, version 1.2015. J Natl Compr Canc Netw. 2015; 13: 194-227. 